Three-dimensional printing for restoration of the donor face : A new digital technique tested and used in the first facial allotransplantation patient in Finland

Makitie, A. A.

2016-12

Makitie , A A , Salmi , M , Lindford , A , Tuomi , J \& Lassus , J 2016 , ' Three-dimensional printing for restoration of the donor face : A new digital technique tested and used in the first facial allotransplantation patient in Finland ' , Journal of Plastic, Reconstructive \& Aesthetic Surgery , vol. 69 , no. 12 , pp. 1648-1652 . https://doi.org/10.1016/j.bjps.2016.09.021

http://hdl.handle.net/10138/229652

https://doi.org/10.1016/j.bjps.2016.09.021

publishedVersion

Downloaded from Helda, University of Helsinki institutional repository.

This is an electronic reprint of the original article.

This reprint may differ from the original in pagination and typographic detail.

Please cite the original version. 


\title{
Three-dimensional printing for restoration of the donor face: A new digital technique tested and used in the first facial allotransplantation patient in Finland
}

\author{
A.A. Mäkitie ${ }^{a, c, d, *}$, M. Salmi ${ }^{a}$, A. Lindford $^{\text {b }}$, J. Tuomi ${ }^{\text {a }}$, \\ P. Lassus ${ }^{b}$
}

a Department of Mechanical Engineering, School of Engineering, Aalto University, Espoo, Finland

b Department of Plastic Surgery, University of Helsinki and Helsinki University Hospital, Helsinki,

Finland

${ }^{\mathrm{c}}$ Division of Ear, Nose and Throat Diseases, Department of Clinical Sciences, Intervention and

Technology, Karolinska Institutet, Karolinska Hospital, SE-17176 Stockholm, Sweden

d Department of Otolaryngology - Head \& Neck Surgery, University of Helsinki and Helsinki University

Hospital, Helsinki, Finland

Received 5 July 2016; accepted 26 September 2016

\section{KEYWORDS \\ 3D printing; \\ Additive \\ manufacturing; \\ Cadaver; \\ 3D scanning; \\ Rapid prototyping; \\ Finland's face \\ transplant}

\begin{abstract}
Summary Background and aims: Prosthetic mask restoration of the donor face is essential in current facial transplant protocols. The aim was to develop a new three-dimensional (3D) printing (additive manufacturing; AM) process for the production of a donor face mask that fulfilled the requirements for facial restoration after facial harvest.

Materials and methods: A digital image of a single test person's face was obtained in a standardized setting and subjected to three different image processing techniques. These data were used for the 3D modeling and printing of a donor face mask. The process was also tested in a cadaver setting and ultimately used clinically in a donor patient after facial allograft harvest.

Results: and Conclusions: All the three developed and tested techniques enabled the 3D printing of a custom-made face mask in a timely manner that is almost an exact replica of the donor patient's face. This technique was successfully used in a facial allotransplantation donor patient.

(C) 2016 British Association of Plastic, Reconstructive and Aesthetic Surgeons. Published by Elsevier Ltd. All rights reserved.
\end{abstract}

* Corresponding author. Dept. of Otolaryngology - Head \& Neck Surgery, Helsinki University Hospital, P.O. Box 263, FI-00029 HUS, Finland. E-mail address: antti.makitie@helsinki.fi (A.A. Mäkitie). 


\section{Introduction}

Experience in facial allotransplantation is accumulating in several centers worldwide. From 2005, there have been 31 reported facial transplants in seven different countries. ${ }^{1}$ These facial transplants have varied from partial to full facial transplants; however, all of them have included a major part of the facial skin and soft tissues. Therefore, in contrast with solid organs, facial procurement leaves a significant visible disfigurement with consequent ethical issues. The approach to facial restoration after facial procurement has varied between different centers internationally and is subject to varying cultural factors. In some cultures, simple coverage of the remnant facial structures with a simple gauze dressing after facial procurement may suffice. However, in other cultures, there is a need for visual restoration after facial procurement. In order to safeguard the dignity of the donor and respect for the family, it is important to restore the facial appearance after procurement. This is particularly the case in "open casket" burials.

In the literature, there is a scarcity of reports on the current available options for donor face mask production. These methods include masks handmade from resin or silicone. ${ }^{2,3}$ In short, a mold is obtained from the donor face using alginate, and the mask is prepared using this negative imprint. Next, a replica is prepared from plaster from which a silicone or resin mask is then made, and the mask is finally refined using makeup application. However, the mask is not a perfect copy of the donor's face. There are many steps involved, and the end result relies on the skills of the anaplastologist who should work in close tandem with the facial harvesting team.

Additive manufacturing (AM) aka three-dimensional (3D) printing refers to the technologies used for printing a 3D object and is currently used in various medical applications. ${ }^{4}$ The combination of computed tomography (CT) and AM can be applied for the production of medical models for preoperative planning. ${ }^{5} \mathrm{AM}$ technologies are utilized for anatomic personalization of prosthetic sockets, ${ }^{6}$ and additive manufactured implants, ${ }^{7,8}$ dental crowns, ${ }^{9}$ and denture frameworks ${ }^{10}$ have also been successfully used. Our earlier experience with these technologies has comprised various dental and maxillofacial applications. ${ }^{11-13}$ Our and other studies have provided validation of the AM concept as a workable solution for various clinical purposes using modern digital technology and obviating the need for a manual working phase.

Here, our aim was to develop and evaluate a new digital process for the manufacturing of a donor face mask for facial restoration using a multidisciplinary setup. We tested three different 3D digital imaging methods for producing a 3D printed donor face mask. Furthermore, this process was investigated in a cadaver setting to evaluate the timing, logistics, and cooperational aspects of the process. This new technique was then used for facial restoration in the first facial allotransplantation donor patient in Finland.
Materials and methods

\section{D digital imaging method testing}

A volunteer provided fully informed consent for the study as a single test person, and digital images of his face were obtained indoors in a standardized setting. These were then manipulated using three different image-processing techniques. These data were used for the 3D modeling and printing of the donor face mask (Table 1).

Two of the methods were based on reconstructing the geometry and colors from photographs (123D, Autodesk Inc., USA and Proface, Planmeca Oy, Finland). The third one used structured light to capture geometry and a monochrome camera with a projector for color channels (red, green, and blue) to capture colors (DAVID-SLS-1 scanning set, DAVID Vision Systems GmbH, Germany).

Data obtained from scanning were then converted to a 3D (Polygon File Format) format using various software (Table 1). Models were repaired, manipulated, and thickened using the 3DataExpert software (3DataExpert 10.0.0.7, DeskArtes Oy, Finland).

The 3D data of the planned mask were then transferred to the $A M$ machine using a ZPrint CAD format. The final physical object was manufactured using a binder jetting method with a full color complement (ZPrinter 450 or 650, 3D Systems Corp, USA).

\section{Cadaver case}

The described process was then tested in a cadaver during a facial procurement practice session performed by the Helsinki Facial Allotransplantation team. The face of the deceased was photographed the day before using a Canon Powershot G12 (Canon Inc., Japan) digital camera in normal lighting at the mortuary. The 123D Catch was not used because the licensing agreement only allows the manufacturer to use created 3D models for marketing purposes. Therefore, Agisoft Photoscan (AgiSoft LLC, Russia) was used instead for creating the 3D model from the photographs. The editing and manufacturing were completed as previously described. The face of the cadaver was dissected as planned for the first facial allotransplantation in Finland and included the full face, soft tissues, and Le Fort II type maxilla. The mask was then inserted after dissection and secured with gauze.

\section{Allotransplantation patient}

This new donor mask-manufacturing technique was then clinically used in Finland's first facial allotransplantation case. The donor patient was photographed using a standard digital camera soon after the decision for facial procurement was confirmed. The same processing as outlined above was conducted concurrently with the multiple organ and facial allograft harvesting operation. The only difference compared with the cadaver test case was that the donor now had an endotracheal tube and some stains on the skin. These were managed by using standard photo-editing 
Table 1 The three different techniques used for mask production.

\begin{tabular}{|c|c|c|c|c|c|c|}
\hline \multirow[t]{2}{*}{ Name } & \multirow{2}{*}{$\begin{array}{l}\text { Digitizing face } \\
\text { Scanner }\end{array}$} & \multirow{2}{*}{$\begin{array}{l}\text { Creating 3D model } \\
\text { from scanned data } \\
\text { Scanning software }\end{array}$} & \multirow{2}{*}{$\begin{array}{l}\text { Manipulating 3D model } \\
\text { Repairing and } \\
\text { thickening }\end{array}$} & \multicolumn{3}{|c|}{ Printing process } \\
\hline & & & & Printer & Powder & $\begin{array}{l}\text { Layer } \\
\text { thickness }\end{array}$ \\
\hline DAVID & $\begin{array}{l}\text { DAVID Structured } \\
\text { Light Scanner } \\
\text { (DAVID Vision Systems } \\
\text { GmbH, Germany) }\end{array}$ & $\begin{array}{l}\text { DAVID-LASERSCANNER } 3.6 .0 \\
\text { (DAVID Vision Systems } \\
\text { GmbH, Germany) }\end{array}$ & $\begin{array}{l}\text { 3DataExpert 10.0.0.7 } \\
\text { (DeskArtes Oy, Finland) }\end{array}$ & $\begin{array}{l}\text { Zprinter } 450 \\
\text { (3D Systems } \\
\text { Corp, USA) }\end{array}$ & ZP 150 & $0.1 \mathrm{~mm}$ \\
\hline $123 D$ & $\begin{array}{l}\text { Canon Powershot G12+ } \\
\text { flash, } 9 \text { photos } \\
\text { (Canon Inc., Japan) }\end{array}$ & $\begin{array}{l}\text { 123D CATCH } \\
\text { (Autodesk Inc., USA) }\end{array}$ & $\begin{array}{l}\text { 3DataExpert 10.0.0.7 } \\
\text { (DeskArtes Oy, Finland) }\end{array}$ & $\begin{array}{l}\text { Zprinter } 450 \\
\text { (3D Systems } \\
\text { Corp, USA) }\end{array}$ & ZP 150 & $0.1 \mathrm{~mm}$ \\
\hline Proface & $\begin{array}{l}\text { Planmeca ProMax ProFace } \\
\text { (Planmeca Oy, Finland) }\end{array}$ & $\begin{array}{l}\text { ProMax ProFace integrated } \\
\text { software (Planmeca } \\
\text { Oy, Finland) }\end{array}$ & $\begin{array}{l}\text { 3DataExpert 10.0.0.7 } \\
\text { (DeskArtes Oy, Finland) }\end{array}$ & $\begin{array}{l}\text { Zprinter } 650 \\
\text { (3D Systems } \\
\text { Corp, USA) }\end{array}$ & ZP150 & $0.1 \mathrm{~mm}$ \\
\hline
\end{tabular}

methods. Following the final detachment of the facial allograft, the manufactured donor face mask was then secured in the correct place.

All work in this study was conducted in accordance with the Declaration of Helsinki (1964).

\section{Results}

\section{D digital imaging method testing}

All the three evaluated techniques were easy to use and proved feasible for successful donor face mask manufacturing. The 123D technique and Proface require the use of a standard digital camera. The process using $123 \mathrm{D}$ was the easiest but did require some manual manipulation. The DAVID technology uses a separate device to acquire the images and required a considerable amount of manual manipulation.

When evaluating the geometry of the mask without colors and visually with colors, the quality of the mask with the 123D technique was the poorest and with Proface was the best (Figure 1). Figure 2 shows images of the final 3D manufactured facial masks and aims to highlight differences such as the physical characteristics of the final mask among the three digital manufacturing techniques. Two projections were chosen to adequately demonstrate the resemblance between the test patient and final facial mask characteristics (Figure 2).

For 123D, Proface, and DAVID, the capture of the image and data transfer took 15-30 min, the data conversion and manipulation took $15 \mathrm{~min}$, and the physical 3D manufacturing took $8 \mathrm{~h}$. The manufacturing cost was estimated to be $400 €$ for each death mask.

\section{Cadaver case}

The process time was $9 \mathrm{~h}$. The mask included the full face and submental area. The mask was of the correct size and could be fitted without any problems. The anatomical areas that were not included in the mask but had been dissected with the face were covered with gauze. The quality of the mask was assessed to be satisfactory for its purpose.

\section{Allotransplantation patient}

Photographs of the donor face were obtained by one of the surgeons $1 \mathrm{~h}$ after the decision for proceeding for face transplantation had been made. The distance between the donor lateral canthal ligaments was measured to enable the technician to make the appropriate scale adjustment of the mask. Because of patient confidentiality, the data were obtained from the hospital and delivered near to the 3D printer within $15 \mathrm{~min}$. The processing of the images and creation of the 3D model took $2.5 \mathrm{~h}$, and subsequent 3D printing with postprocessing took $12.5 \mathrm{~h}$. The delivery of the new donor face mask to the donor patient's hospital took $15 \mathrm{~min}$, and the entire process took $15.5 \mathrm{~h}$. The mask now included the neck area in addition to the full face. The mask promptly arrived following the detachment of the facial allograft and could be easily secured by drill holes to the remaining facial structures. The appearance was remarkable in its resemblance to the donor patient.

\section{Discussion}

$A M$ is increasingly being used in medical applications. We initially tested $A M$ as an approach to fabricate donor face masks in preparation for facial restoration in the donor patient in a facial allotransplantation program. In contrast to the conventional impression techniques using an impression medium such as alginate, the production of an AM mask is based on the imaging of the donor. ${ }^{14}$ This new technique was then successfully applied clinically to the first facial allotransplantation case in Finland.

In the present study, the initial imaging data were obtained using three different techniques, and all the three manufactured masks proved to have sufficient quality for the required purpose. However, the DAVID system requires a separate device, and Proface is dependent on the CT imaging system. Because the standard digital camera (123D) technique was the easiest to use and provided a feasible donor face mask, it was chosen for testing in the 


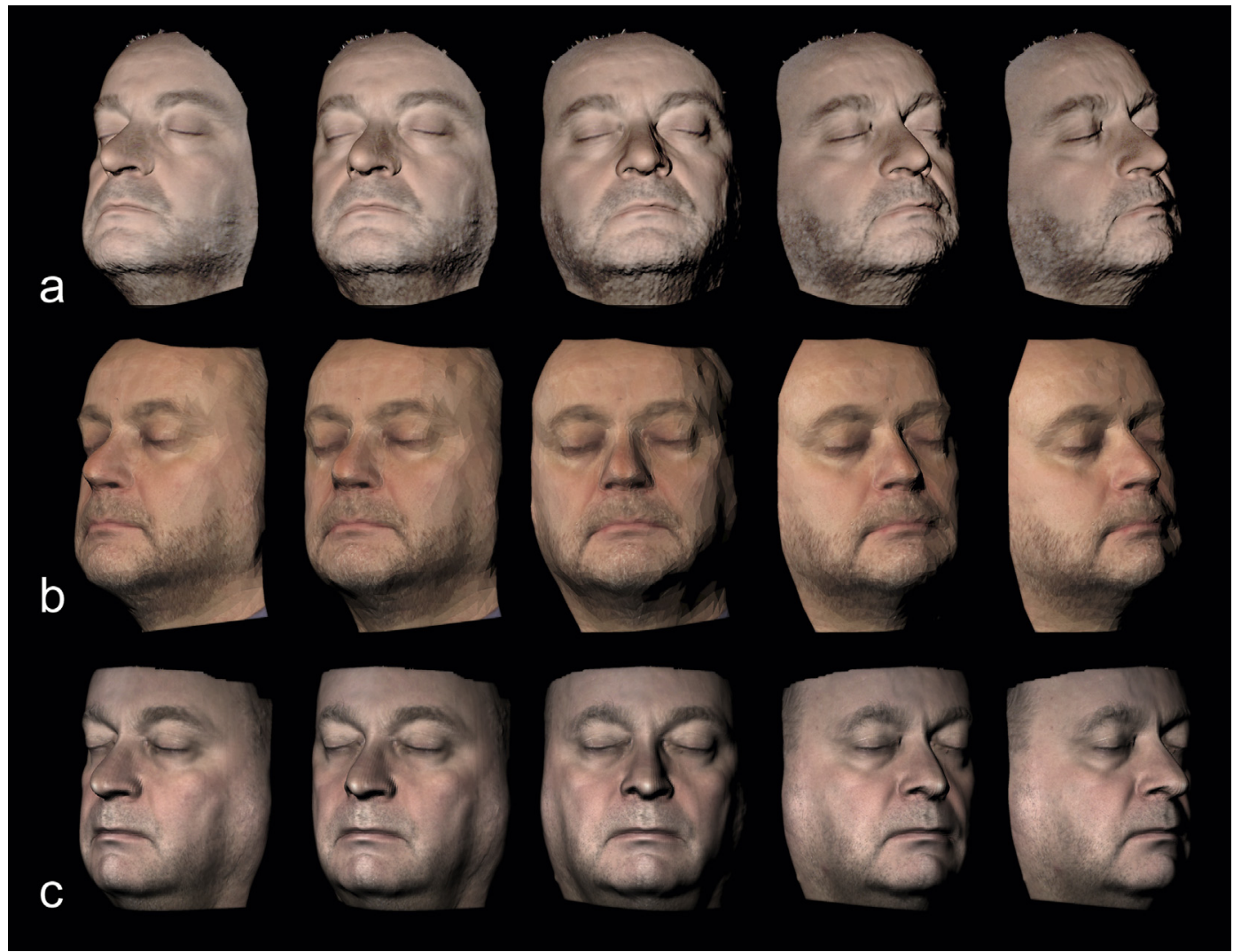

Figure 1 Three-dimensional (3D) images of the test person using the three techniques: a) DAVID, b) 123D, and c) Proface.

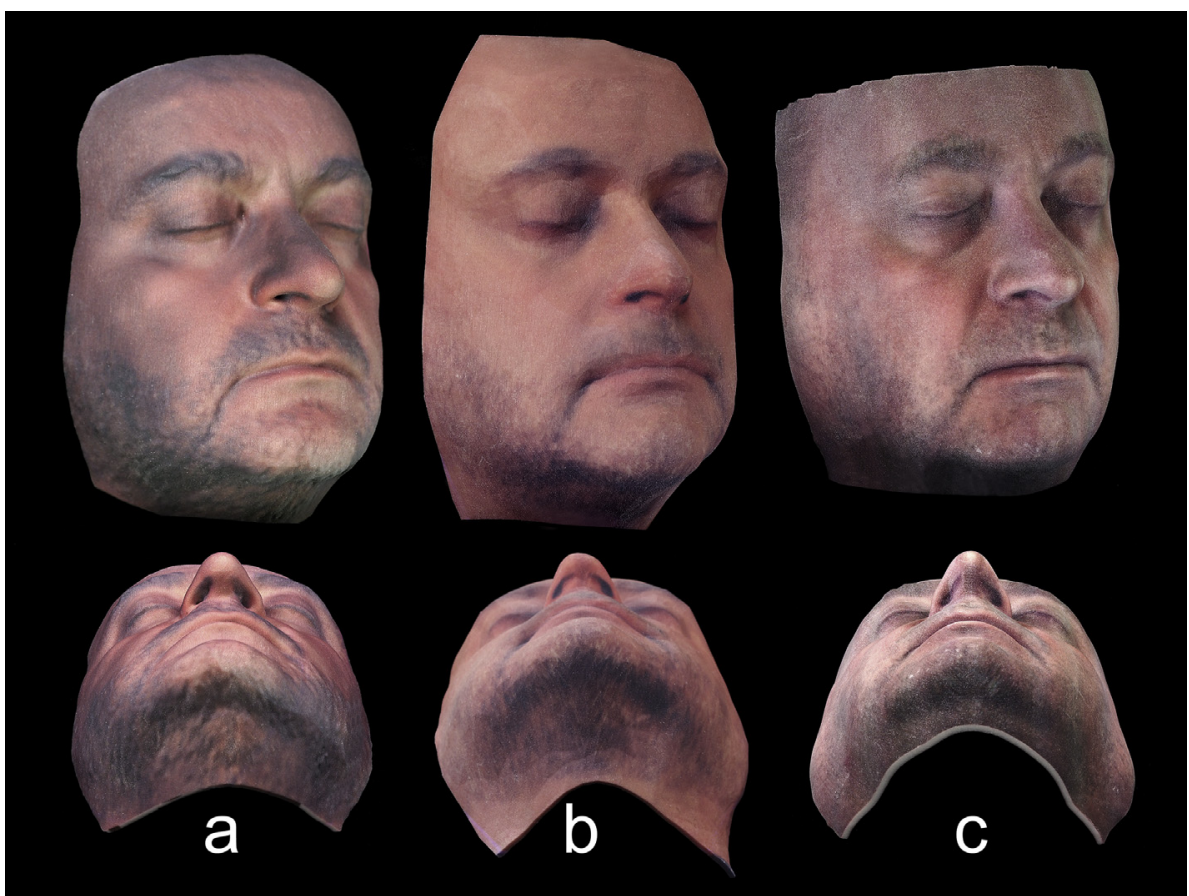

Figure 2 Pictures of 3D printed facial masks of the test person: a) DAVID, b) 123D, and c) Proface.

cadaver setting. After being confirmed to provide a good quality mask in the cadaver case, the digital camera was consequently selected for use in the forthcoming face transplant case. It had been proven to provide enough digital data for an anatomical and a realistic replica of the donor face. For the manipulation and production of the mask, a separate technician is indeed required, as is the case with the conventional impression mold processes. ${ }^{2,3}$ 
The technician does not have to travel with the harvesting team. However, an on-call system needs to be organized for nights and weekends, and the mask has to be delivered to the donor patient's hospital.

In our study setting, the Proface system produced the best quality mask. Nevertheless, the masks made by all the three methods were almost exact copies of the original face (Figure 2) and fulfilled the criteria for the mask. The mask material is not elastic; thus, its texture is not natural, which is also the case with handmade masks. It is also possible to secondarily shape the mask if needed; in addition, drill holes can be made to secure it to the facial structures of the donor patient. In cases of partial face procurement, the mask can be shaped to fit the defect.

The time duration of donor mask manufacturing for all three techniques varied between 9 and $15 \mathrm{~h}$. This did not include the time required to deliver the mask to the donor patient location. However, the image of the donor can be obtained immediately after the decision for procurement has been made and well before the commencement of the donor operation so that it is possible to have the mask ready once the face has been harvested.

The calculated costs for a donor face mask are dependent on the required equipment and personnel costs. There is a need for a digital camera and surface or CT scanner, and these are available in most medical institutions. The work-related costs, however, are much less than for handmade masks because most of the production is automated. In the actual facial allotransplantation case, the costs incurred were estimated to be approximately $1500 €$.

\section{Conclusions}

A new process was developed and tested for the production of a novel donor face mask that fulfilled the requirements of facial restoration after facial harvest. All the three techniques enabled the 3D printing of a custom-made donor face mask in a timely manner that is almost an exact replica of the donor's face. These findings were confirmed by the successful use of this process in Finland's first facial allotransplantation case. We found a standard digital camera feasible to be used in this setting. These techniques can be adjusted in terms of imaging equipment and timeline according to each individual program.

\section{Ethical approval details}

This study was approved by the Research Ethics Board of Helsinki University Hospital.

\section{Funding statement}

See Acknowledgements.

\section{Declaration of conflicting interests}

The authors declare no conflicting interest.

\section{Acknowledgements}

The authors would like to thank the Helsinki University Hospital Research Fund (TYH2014234), the Sigrid Jusélius Foundation, and Tekes, the Finnish Funding Agency for Technology and Innovation (Dnro 660/31/2010) for financing this research and Planmeca Oy for collaboration. This study was part of the MedAMan research project. We also thank Pekka Paavola, M.Sc. (Tech), for the photographs.

\section{References}

1. Roche NA, Blondeel PN, Van Lierde KM, et al. Facial transplantation: history and update. Acta Chir Belg 2015;115: 99-103.

2. Renshaw A, Teerathraj C, Clarke A, et al. An artificial prosthesis to reconstruct donor defects following facial transplantation. Clin Transpl 2007;21:574-6.

3. Quilichini J, Hivelin M, Benjoar MD, et al. Restoration of the donor after face graft procurement for allotransplantation: report on the technique and outcomes of seven cases. Plast Reconstr Surg 2012;129:1105-11.

4. Tuomi J, Paloheimo KS, Vehviläinen J, et al. A novel classification and online platform for planning and documentation of medical applications of additive manufacturing. Surg Innov 2014;21:553-9.

5. Sanghera B, Naique S, Papaharilaou Y, et al. Preliminary study of rapid prototype medical models. Rapid Prototyp J 2001;7: 275-84.

6. Ng P, Lee PSV, Goh JCH. Prosthetic sockets fabrication using rapid prototyping technology. Rapid Prototyp J 2002;8:53-9.

7. Poukens J, Laeven P, Beerens $M$, et al. A classification of cranial implants based on the degree of difficulty in computer design and manufacture. Int J Med Robotics Comput Assisted Surg 2008;4:46-50.

8. Salmi M, Tuomi J, Paloheimo KS, et al. Patient-specific reconstruction with 3D modeling and DMLS additive manufacturing. Rapid Prototyp J 2012;18:209-14.

9. Tara MA, Eschbach S, Bohlsen F, et al. Clinical outcome of metal-ceramic crowns fabricated with laser-sintering technology. Int J Prosthodont 2011;24:46-8.

10. Williams RJ, Bibb R, Eggbeer D, et al. Use of CAD/CAM technology to fabricate a removable partial denture framework. J Prosthet Dent 2006;96:96-9.

11. Huotilainen E, Jaanimets R, Valášek J, et al. Inaccuracies in additive manufactured medical skull models caused by the DICOM to STL conversion process. J Craniomaxillofac Surg 2014;42:259-65.

12. Salmi M, Paloheimo KS, Tuomi J, et al. A digital process for additive manufacturing of occlusal splints: a clinical pilot study. J R Soc Interface 2013;24:1-6.

13. Salmi M, Tuomi J, Sirkkanen R, et al. Rapid tooling method for soft customized removable oral appliances. Open Dent J 2012; 6:85-9.

14. Grant GT, Liacouras P, Santiago GF, et al. Restoration of the donor face after facial allotransplantation: digital manufacturing techniques. Ann Plast Surg 2014;72:720-4. 